\title{
Effect of apple food matrix on plasma flavan-3-ols distribution and nutrigenomic profile in response to a nutritional challenge in minipigs
}

\author{
Laurent-Emmanuel Monfoulet ${ }^{1}$, Caroline Buffiere ${ }^{1}$, Geoffrey Istas ${ }^{2}$, Claire Dufour ${ }^{3}$, \\ Carine le Bourvelec ${ }^{3}$, Sylvie Mercier ${ }^{1}$, Dominique Bayle ${ }^{1}$, Céline Boby ${ }^{4}$, Didier Remond ${ }^{1}$, \\ Patrick Borel $^{5}$, Ana Rodriguez-Mateos ${ }^{6}$, Dragan Milenkovic ${ }^{1}$ and Christine Morand ${ }^{1}$ \\ ${ }^{1}$ Université Clermont Auvergne, INRA, UNH, Unité de Nutrition Humaine, Clermont-Ferrand, France, \\ ${ }^{2}$ Department of Nutritional Sciences, King's College, London, United Kingdom, \\ ${ }^{3} I N R A$ Sécurité \& Qualité des Produits d'Origine Végétale - UMR408 Université d'Avignon, Avignon, France, \\ ${ }^{4}$ INRA, Unité Mixte de Recherches sur les Herbivores, Clermont-Ferrand, France, \\ ${ }^{5}$ INRA, UMR 1260 INRA/1062 INSERM/Université Aix-Marseille, Marseille, France and \\ ${ }^{6}$ Department of Nutritional Sciences, King's College, London, France
}

\begin{abstract}
Food matrix is known to interact with some dietary constituents and microconstituents during digestion. These interactions may potentially affect the metabolism and bioavailability of some compounds, and as a consequence modulate their biological effects. In this context, the aim of this study was to determine the effect of apple food matrix on the bioavailability of flavan-3-ols and on the ability of these compounds to modulate the nutrigenomic response to a high fat challenge in minipigs.

Adult male Yucatan minipigs $(n=5)$ were assigned to a random treatment sequence of high-fat meals non supplemented or supplemented with $250 \mathrm{~g}$ of raw apple, $250 \mathrm{~g}$ of apple puree or $1.4 \mathrm{~g}$ of apple polyphenols extract, with a 7-days washout period between each treatment. Each supplementation provided $155 \mathrm{mg}$ flavan-3-ol monomers. At each treatment period, fasting- and 1h-, $2 \mathrm{~h}-$, 3h-postprandial blood samples were collected, and the concentration in flavan-3-ol monomers was measured on hydrolyzed serum, using UPLC-Q-TOF MS. The ability of apple-derived products to modulate the postprandial gene expression profile was assessed and compared in circulating PBMCs collected at $3 \mathrm{~h}$ after consumption of the four tested meals using a microarray analysis.

Results show that the apple matrix did not affect the kinetic of the postprandial absorption of flavan-3-ol monomers. The total flavan-3-ols concentrations measured at peak were significantly higher in the extract (x1.75), suggesting an impact of the apple matrix on flavan-3-ols absorption. However, no significant difference in total flavanols was observed between raw apple and apple puree.

Principal Component Analysis of the microarray data from PBMCs identified three distinct clusters of gene expression patterns: one corresponding to gene expression profiles after the high-fat meal, one for meal supplemented with raw apples or apple puree, and a third cluster for meal supplemented with polyphenol extract. A set of 309 genes was identified as differentially expressed by applederived products compared to high-fat meal alone, including 93 modulated with the three apple products. The variations in gene expression were similar for only $75 \%$ of the 93 genes, suggesting that the apple matrix affects the nutrigenomic response to flavan-3-ols. A bioinformatics analysis revealed that genes affected by apple-derived products are involved in inflammation and leukocyte transendothelial migration, suggesting a beneficial impact of apple-derived products.

In conclusion, these results raise awareness for considering the impact of food matrix on the biological responsiveness of polyphenols in future nutritional studies.
\end{abstract}

\section{Conflict of Interest}

There is no conflict of interest 